\title{
Assessment of dry eye status in type 2 diabetic patients in tertiary health care hospital, India
}

\author{
Ibtesam Nasimul Hasan ${ }^{1}$, Pratik Aggarwal ${ }^{1}$, Akshata Gurav ${ }^{1}$, Nilay Patel $^{1}$ \\ ${ }^{1}$ Junior Resident, Dr. DY Patil Hospital and Research Centre, Nerul, Navi Mumbai
}

\begin{abstract}
:
Objective: (1) Assessment of incidence of dry eyes in type 2 diabetic patients. (2) To determine the association of Diabetic Retinopathy with dry eyes.

Material and Methods: A cross-sectional study consisting of 100 Type 2 diabetic patients was carried out in Dr. D. Y. Patil Hospital \& Research Centre, Nerul, Navi Mumbai during 2012-2014. After applying inclusion and exclusion criteria 100 diabetic patients who attended the out-patient department of the Department of Ophthalmology, were selected.

Results: Mild form of dry eyes was most common (20\%). There was no significant association of age, sex and duration of diabetes with incidence of dry eyes. Abnormal tear break up time was abnormal in 33\%. Abnormal Fluorescein staining was seen in $20 \%$ study subjects. Moderate non-proliferative diabetic retinopathy was significantly more common in diabetic patients with dry eyes.

Conclusion and Recommendation: Examination of dry eyes should be an integral part of the assessment of diabetic eye disease so as to improve the patient's comfort and to prevent or minimize further structural damage to the ocular surface.
\end{abstract}

Keywords: Dry eyes, type 2 diabetes, retinopathy

\section{Introduction}

Diabetes is one of the leading health related catastrophes the world has ever witnessed. ${ }^{1}$ It affects millions of people all over the world. WHO has labeled India as "The diabetic capital of the world" as it has the highest number of diabetics in the world. It estimates that there will be 370 million people with diabetes on the planet by 2030, which is nearly twice the figure reported in $2000 .^{2}$

Diabetes is often associated with several ocular conditions mainly diabetic retinopathy, cataracts, refractive errors, nerve palsies, glaucoma, hordeolosis, macular edema, neovascular glaucoma and orbital infections. However, one of the most common, but often neglected complication associated with diabetes is dry eye. Diabetic patients often complain of burning or foreign body sensation indicating a clear role of tear film abnormality. These patients are also more prone to corneal ulcerations, superficial punctate keratopathy and persistent epithelial defects.

Few numbers of studies have been done regarding the tear film abnormalities in diabetics and decrease in tear production has been reported, but the overall data is not conclusive. Moreover, the ocular surface examination is usually ignored in diabetics and much importance is given to Diabetic retinopathy in routine practice.

Hence the present study was undertaken to evaluate the amount of tear production, the stability of the tear film and the condition of the ocular surface in diabetic individuals in order to detect possible tear film abnormalities and its association with diabetic retinopathy. This would improve the patient's comfort and prevent or minimize further structural damage to the ocular surface.

\section{Research Method and Methodology}

Present cross-sectional study consisting of 100 Type 2 diabetic patients was carried out to assess the presence of dry eyes and to investigate the relationship of retinopathy with dry eyes. After applying inclusion and exclusion criteria 100 diabetic patients who attended the out-patient department of the Department of Ophthalmology, Dr. D. Y. Patil Hospital \& Research Centre, Nerul, Navi Mumbai during 2012-2014.

\subsection{Inclusion criteria}

All patients of either sex, in all age groups, diagnosed to have Diabetes Mellitus Type 2 of any duration. 


\subsection{Exclusion criteria}

i. Patients with systemic diseases and local ocular disease/surface abnormalities as assessed by history and clinical examination, other than diabetes mellitus, which are known to cause dry eyes/ocular surface abnormalities.

ii. Patients who were chronic contact lens wearer.

iii. Patients who have had undergone ocular surgeries in the past.

iv. Patients on local or systemic medications, which are known to cause dry eyes/ocular surface disorders.

After taking informed consent, detailed history regarding patients name, age, sex, occupation, address, presenting symptoms, duration, progression, and associated conditions were recorded. Detailed history regarding diabetes such as type of diabetes, duration, type of treatment, overall control in the past three months (based on sugar levels, HbAlc values if available), FBS and PPBS levels were recorded.

A validated eight item questionnaire of ocular symptoms relating to dry eye was used which included the following questions:

1. Do your eyes ever feel dry?

2. Do you ever feel a gritty or sandy sensation in your eye?

3. Do your eyes ever have a burning sensation?

4. Are your eyes ever red?

5 . Do your eyes ever feel sticky?

6. Do your eyes ever feel watery or tearing?

7. Do you notice much crusting on your lashes?

8. Do your eyes ever get stuck shut?

Presence of a symptom from the dry eye questionnaire was graded as:

- Rarely (atleast once in 3-4 months),

- Sometimes (once in 2-4 weeks),

- Often (at least once a week), or

- All the time.

Presence of one more symptoms often or all the time was taken as positive

A brief general and systemic examination was carried out.

Ocular examination included recording visual acuity with Snellen's chart (in patients with visual acuity less than 6/60, acuity was recorded as counting fingers at particular distance or hand movements or perception of light or projection of rays).

\section{Examination}

Detailed anterior segment examination was done under slit lamp. Condition of lids, conjunctival surface (dryness, wrinkling, sheen) and corneal surface was noted.

Cornea was evaluated in detail for its sheen, surface (superficial punctate keratitis (SPK)/mucous plaques/filamentary keratitis). Sensation was recorded after schirmer'stest with a fine moist cotton wisp and graded as normal, reduced or absent.

\section{Tear film evaluation was done in the following order:}

- Tear meniscus height was recorded as normal or low (under slit lamp, thin beam)

- Pre-corneal tear film was observed for presence of debris (mucous/oil droplets/debris)

\section{Tear break up time measurement}

No anesthesia was used. A dry fluorescein strip is touched to the inferior fornix with the patient looking up. The cornea scanned under low slit lamp magnification using blue cobalt filtered light. The patient was instructed to blink once or twice and then stare straight ahead without blinking. The time of appearance of the first dry spot formation (small black spots within the blue-green field) from the last blink measured the tear film BUT. Values $<10$ seconds were taken as abnormal.

Fluorescein staining of cornea was graded from $0-3$

0 - No staining of corneal epithelial surface.

1 - Mild staining occupying $<1 / 3$ of corneal epithelial surface.

2 - Moderate staining occupying $<1 / 2$ of corneal epithelial surface.

3 - Severe staining occupying $>1 / 2$ of the corneal epithelial surface. 
Schirmer's test without Anaesthesia(basal and reflex tearing)

It was performed by placing a precut strip of filter paper in the inferior cul-de-sac; Patient was asked to blink normally, and the amount of wetting of the paper strip after 5 minutes was measured. Wetting of $\leq 10 \mathrm{~mm}$ was taken as abnormal.

\section{Schirmer's test with Anaesthesia(basal tearing)}

The basal secretion test is performed following the instillation of topical anesthetic (Paracain Proparacainehydrochloride drops) and the placement of a thin strip of filter paper in the inferior cul-de-sac. Measurement of $\leq 5 \mathrm{~mm}$ was taken as abnormal, $5-10 \mathrm{~mm}$ as equivocal

Dry eye was having one or more symptoms (often or all the time present) along with one or more positive clinical findings (based on slit lamp examination) and one or more positive clinical tests (tear break up time of $\leq 10$ seconds, schirmer's test score $\leq 10 \mathrm{~mm}$, with anesthesia $\leq 5 \mathrm{~mm}$, fluorescein score of $\geq 1$. Asymptomatic patients with positive signs or positive tests were also considered in the diagnosis.

Dry eye was graded into three types-mild, moderate, and severe.

- Mild dry eye can be defined in patients who have a Schirmer's test of less than $10 \mathrm{~mm}$ in 5 minutes, T BUT less than 10 seconds and less than one quadrant of staining of the cornea.

- Moderate dry eye can be defined in a Schirmer's test of 5 to $10 \mathrm{~mm}$ in 5 minutes, T BUT of 5 to 10 seconds with punctate staining of more than one quadrant of the corneal epithelium.

- Severe dry eye can be defined as diffuse punctate or confluent staining with fluorescein of the corneal epithelium, often with filaments and diffuse punctate or confluent staining of the conjunctival epithelium. The Schirmer'svalues in these patients is less than $5 \mathrm{~mm}$ in 5 minutes and T BUT less than 5 seconds.

Intraocular pressure [Schiotz method] was recorded as a part of routine ocular examination.

Detailed fundus examination [under mydriasis] was done under direct and indirect ophthalmoscopy and $90 \mathrm{D}$ slit lamp examination.

Retinopathy if present was classified as:

-Non-Proliferative Diabetic Retinopathy (NPDR)

-Mild- NPDR, Moderate-NPDR, Severe- NPDR

-Proliferative Diabetic Retinopathy

-Early PDR, High risk PDR

The collected data was numerically coded and entered in Microsoft Excel 2007 and then transferred to SPSS version 20.0 Added data was analyzed with appropriate test. The comparison of Quantitative variables between and within the groups was done using student's t- test, while the Qualitative data was compared using chi-square test. The confidence limit for significance was fixed at $95 \%$ level with $\mathrm{p}$-value $<0.05$.

\section{Results}

Table 1 describes that in this study out of 100 Diabetic patients, 42 had dry eyes. Mild form of dry eyes was most common (20\%). Table 2 reveals the association of age, sex and duration of diabetes with incidence of dry eye. Majority of the patients were between the ages of 40-60 years. There was no significant association of age, sex and duration of diabetes with incidence of dry eyes.

Table 1: Incidence of dry eye (n-100)

\begin{tabular}{|l|c|}
\hline Dry eyes status & No. $(\%)$ \\
\hline No dry eyes & $58(58)$ \\
\hline Mild dry eyes & $20(20)$ \\
\hline Moderate dry eyes & $16(16)$ \\
\hline Severe dry eyes & $6(6)$ \\
\hline
\end{tabular}

Table 2: Association of incidence of dry eyes with age, sex and duration of diabetes

\begin{tabular}{|l|l|c|c|c|c|}
\hline \multicolumn{2}{|c|}{} & Count & Dry eyes & P-value & OR (Dry eyes) \\
\hline \multirow{4}{*}{ Sex } & Male & 48 & 21 & 0.733 & 1.15 \\
\cline { 2 - 6 } & Female & 52 & 21 & 0.733 & 1.15 \\
\hline \multirow{5}{*}{ Age in years } & $<-20$ & 0 & 0 & & - \\
\cline { 2 - 6 } & $21-30$ & 1 & 0 & 0.392 & 0.580 \\
\cline { 2 - 6 } & $31-40$ & 12 & 8 & 0.065 & 0.972 \\
\cline { 2 - 6 } & $41-50$ & 27 & 11 & 0.914 & 1.1208 \\
\cline { 2 - 6 } & $51-60$ & 21 & 12 & 0.479 & 1.130 \\
\cline { 2 - 6 } & $61-70$ & 7 & 3 & 0.683 & 0.978 \\
\cline { 2 - 6 } & $71-80$ & 1 & 0 & 0.962 & - \\
\cline { 2 - 6 } & $>80$ & & & & \\
\hline
\end{tabular}


Assessment of dry eye status in type 2 diabetic patients in tertiary health care hospital, India

\begin{tabular}{|l|l|c|c|c|c|}
\hline \multirow{4}{*}{$\begin{array}{l}\text { Duration of } \\
\text { Diabetes }\end{array}$} & $\leq 6$ months & 13 & 5 & 0.514 & 1.106 \\
\cline { 2 - 6 } & $6-12$ months & 6 & 3 & 0.498 & 0.83 \\
\cline { 2 - 6 } & $1-5$ years & 37 & 15 & 0.498 & 0.909 \\
\cline { 2 - 6 } & $5-10$ years & 19 & 10 & 0.216 & 0.751 \\
\cline { 2 - 6 } & $10-20$ years & 21 & 7 & 0.258 & 1.329 \\
\cline { 2 - 6 } & $>20$ years & 4 & 2 & 0.561 & 0.833 \\
\hline
\end{tabular}

Table 3 shows that significantly more fasting and postprandial blood sugar in diabetic patients with dry eyes. Meibomitis and reduced corneal sensation were significantly more common among diabetics with dry eyes (Table 4). Symptoms like redness, watering, stickiness, crusting etc were significantly more common among diabetic patients with dry eyes (Table 5). Table 6 shows clinical signs of dry eyes in diabetic patients. $27 \%$ of the patients had low tear meniscus and conjunctival abnormalities that included dull and hyperemic congested conjunctiva.

Table 3: Mean blood sugar level

\begin{tabular}{|l|c|c|c|c|}
\hline Glycemic control & No dry eyes & Dry eyes & T-test & P-Value \\
\hline FBS in $\mathrm{mg} / \mathrm{dl}$ & $83.90 \pm 12.294$ & $179.63 \pm 57.28$ & 18.524 & $<0.05$ \\
\hline PPBS in $\mathrm{mg} / \mathrm{dl}$ & $116.15 \pm 10.49$ & $247.75 \pm 88.39$ & 16.709 & $<0.05$ \\
\hline
\end{tabular}

Table 4: Other causes for dry eyes (n-100)

\begin{tabular}{|l|c|c|c|c|}
\hline Other causes for dry eyes & Count & Dry eyes & P-value & OR (Dry eyes) \\
\hline Blepharitis (lids) & 3 & 2 & 0.379 & 0.619 \\
\hline Meibomitis & 6 & 6 & 0.007 & - \\
\hline Reduced corneal sensation & 29 & 20 & $<0.01$ & 0.449 \\
\hline LASER & 10 & 4 & 0.893 & 1.056 \\
\hline Hypertension & 32 & 15 & 0.498 & 0.847 \\
\hline
\end{tabular}

Table 5: Symptoms among study subject (n-100)

\begin{tabular}{|l|c|c|c|c|}
\hline Symptoms & Count & Dry eyes & P-value & OR (Dry eyes) \\
\hline Eye feel Dry & 6 & 6 & 0.003 & - \\
\hline Gritty feeling & 13 & 9 & 0.033 & 3.682 \\
\hline Burning Sensation & 12 & 6 & 0.549 & 1.44 \\
\hline Stickiness & 6 & 5 & 0.034 & 7.703 \\
\hline Watering & 11 & 5 & 0.806 & 1.171 \\
\hline Redness & 5 & 5 & 0.007 & - \\
\hline Crusting & 4 & 4 & 0.016 & - \\
\hline Eyes getting stuck & 5 & 5 & 0.007 & - \\
\hline
\end{tabular}

Table 6: Signs among study subjects (n-100)

\begin{tabular}{|l|c|}
\hline Signs & No. $(\%)$ \\
\hline Low Tear Meniscus & $27(27)$ \\
\hline Abnormal Precorneal tear film & $20(20)$ \\
\hline Conjunctival abnormalities & $27(27)$ \\
\hline Dull Cornea & $23(23)$ \\
\hline
\end{tabular}

Table 7 reveals the results of various tests results. Abnormal tear break up time was abnormal in $33 \%$. Abnormal Fluorescein staining was seen in 20\% study subjects. Schirmer's test with Anesthesia is a better test in terms of sensitivity, specificity, Positive Predictive Value, Negative Predictive Value and accuracy followed by Fluorescein staining and then Tear break up time (Table 8).

Table 7: Various tests in study subjects

\begin{tabular}{|l|c|c|}
\hline Tests & Normal & Abnormal \\
\hline Tear Break up time & 67 & 33 \\
\hline Schirmer's Test without Anaesthesia & 84 & 16 \\
\hline Schirmer'sTest with Anaesthesia & 93 & 7 \\
\hline Fluorescein stain & 80 & 20 \\
\hline
\end{tabular}

Table 8: Diagnostic Statistics of various tests

\begin{tabular}{|l|c|c|c|c|}
\hline $\begin{array}{l}\text { Diagnostic } \\
\text { Statistics }\end{array}$ & $\begin{array}{c}\text { Schirmer's } \\
\text { test without Anaesthesia }\end{array}$ & $\begin{array}{c}\text { Schirmer's test with } \\
\text { Anaesthesia }\end{array}$ & $\begin{array}{c}\text { Tear break } \\
\text { Up time }\end{array}$ & $\begin{array}{c}\text { Fluorescein } \\
\text { stain }\end{array}$ \\
\hline Sensitivity & 32.14 & 100 & 100 & 100 \\
\hline Specificity & 6.25 & 89.23 & 63.04 & 74.36 \\
\hline PPV & 64.29 & 83.33 & 19.05 & 52.38 \\
\hline NPV & 1.72 & 100 & 100 & 100 \\
\hline Accuracy & 26.1 & 93.14 & 70.5225 & 81.665 \\
\hline Kappa & 0.075 & 0.053 & 0.084 & 0.083 \\
\hline
\end{tabular}


Table 9 describes the association of retinopathy with dry eyes. Moderate non-proliferative diabetic retinopathy was significantly more common in diabetic patients with dry eyes.

Table 9: Association of retinopathy with dry eyes

\begin{tabular}{|l|c|c|c|}
\hline Retinopathy(n=100) & Count & Dry eyes & P-value \\
\hline No retinopathy & 63 & 22 & 0.61 \\
\hline Mild Non Proliferative Diabetic Retinopathy (NPDR) & 13 & 6 & 0.754 \\
\hline Moderate Non Proliferative Diabetic Retinopathy & 8 & 6 & 0.049 \\
\hline Severe Non Proliferative Diabetic Retinopathy & 2 & 1 & 0.817 \\
\hline Proliferative Diabetic Retinopathy (PDR) & 13 & 7 & 0.357 \\
\hline Severe Proliferative Diabetic Retinopathy & 1 & - & 0.395 \\
\hline
\end{tabular}

\section{Discussion}

Dry eye syndrome is very common among the general population with $28 \%$ of the adults having dry eye syndrome. ${ }^{3}$ The term "dry eye" may be applied to three different conditions (i) The symptom is ocular dryness (ii) The syndrome is the group of associated clinical manifestations: itching, redness, foreign body sensation, photophobia, and blepharospasm (iii) A large variety of diseases are associated with dry eye and include blink disorders, trachoma and blepharitis. ${ }^{4}$

In our study, out of the 100 patients who participated, $42 \%$ were suffering from dry eye syndrome. According to Beaver Dam Eye Study, $19.8 \%$ of Type II Diabetes had dry eyes. ${ }^{5}$ Seifart and associates demonstrated that diabetic patients had an increased rate of Keratoconjunctivitis sicca, which may be attributed to decreased corneal sensitivity, neuropathy involving innervation of lacrimal glands and loss of goblet cells. Among the type II diabetic patients, $70 \%$ had proven dry eye syndrome. ${ }^{6}$ In a cohort study on 3722 patients, Moss et al showed $18.1 \%$ of diabetics had dry eyes. ${ }^{5}$ Nepp et al showed $43 \%$ of diabetics having dry eyes in his study. ${ }^{7}$ Hom and De Land showed that $52.9 \%$ of patients with either diabetes or borderline diabetes had selfreported clinically relevant dry eyes. ${ }^{8}$

The prevalence of dry eyes has been seen to affect females more than males. ${ }^{9}$ Also, women who used hormone replacement therapy (HRT) had a $69 \%$ greater risk of developing dry eye syndrome. ${ }^{10}$ Moss et al showed a $16.7 \%$ incidence of dry eyes in diabetic women compared to $11.4 \%$ in diabetic men. ${ }^{11}$ In present study, though not significant, incidence of dry eye was more in males compared to females. This similarity could be as we had ruled out women under the treatment of HRT.

With Age, the dry eye symptoms increase as a result of decrease in tear production and flow and increase in evaporation. Kaiserman et al. have reported that the prevalence of dry eye increases with age. ${ }^{12}$ One study suggests that it is increased evaporation and subsequent increase in tear film osmolarity with age that is the more important determinant for dry eyes. ${ }^{13}$ This further suggests meibomian gland dysfunction as the underlying etiologic factor. In our present study, increase in age is associated with dry eyes with $39 \%$ of the population in the age group of 31-70 years having dry eyes. The probability of dry eyes in more than 80 years couldn't be considered due to decrease in number of patients in that age group. According to Scultz et al, autonomic dysfunction may be another cause for prevalence of dry eyes with increase in age. ${ }^{14}$

The prevalence of diabetic microvascular complications is higher in patients with longer duration of diabetes. ${ }^{7}$ These individuals are at an increased risk of developing dry eye syndrome. Seifart and associates demonstrated that diabetic patients had an increased rate of Keratoconjunctivitis sicca, which may be attributed to decreased corneal sensitivity, neuropathy involving innervation of lacrimal glands and loss of goblet cells. ${ }^{6}$ In our present study, no such association was seen, with $37 \%$ patients having dry eyes within the duration of 1 5years.

In our study, elevated FBS and PLBS were found to be associated with dry eyes in the study, indicating the role of hyperglycemia. This could result in high extracellular fluid osmolarity disturbing the tear production. Kaiserman and associates have reported that good blood sugar regulation is important for prevention and control of dry eye syndrome among diabetic patients. ${ }^{12}$

Blepharitis and Meibomitis causes dysfunction of meibomian glands and the lipid component of the tear film causing increase in tear evaporation and hence leading to dry eyes. Also, meibomitis may cause sufficient conjunctival inflammation to decrease tear secretion by damaging accessory lacrimal gland tissue in the conjunctiva. ${ }^{15}$ In our study, 6 patients of diabetes had meibomitis, out of which all six had dry eyes and three patients had blepharitis, out of which two had dry eyes.

The diminished corneal sensitivity in diabetes would play the same pathogenesis as diabetic neuropathy. There is a significant association between dry eyes and reduced corneal sensation. Diabetes mellitus causes accumulation of sorbitol by the action of aldose reductase on excess glucose contributing to the alteration in epithelium and endothelium and thus causing corneal hypoasthaesia. ${ }^{16}$ In our study, 29 patients had reduced corneal sensation, out of which 20 had dry eyes showing significant association. 
Laser and Hypertension were not significantly associated with dry eyes in our study. Although diabetic patients undergone laser are 1.056 times more likely to develop dry eyes. Thirty percent of patients with diabetes had dry eye symptoms out of which eyes feeling dry, redness and eyes getting stuck were the most frequent symptoms. This could be due to associated meibomitis causing increase in tear evaporation. Structural, metabolic and functional abnormalities are found in the conjunctiva and cornea of diabetic patients. These abnormalities may be responsible for clinical corneal manifestation of diabetes. The tear film has also been reported to be unstable. ${ }^{17}$

In evaluating the diagnostic tests for dry eyes, Tear Film Break - Up Time test was said to be a very non-specific test for determination of tear film stability. ${ }^{1}$ Large variations in the same patient has been noticed. T-BUT $<10$ seconds was seen in $67 \%$ of our diabetic patients.

Schirmer's test has been the standard test to measure the tear production. Goebbel has reported that the Schirmer's test reading is significantly reduced among diabetics. In our study, Schirmer's test without anaesthesia showing the total tear secretion was $<10 \mathrm{~mm}$ in $16 \%$ and Schirmer's test with anaesthesia showing the basal tear secretion was $<5 \mathrm{~mm}$ in $7 \%$. Reflex tearing was more affected in our study possibly due to decreased corneal and conjunctival sensitivity, which has been demonstrated in diabetics by electronic aesthesiometry. ${ }^{18}$

Fluorescein stain assessing ocular surface damage was positive in around $20 \%$ of our patients. When these diagnostic tests were statistically analysed, Schirmer's test with anaesthesia was found to be more accurate followed by Fluorescein staining and then tear break up time. According to a study in Spain, diabetic retinopathy patients without subjective symptoms of dry eye and normal Schirmer's and BUT test showed pathological grades of squamous metaplasia. ${ }^{19}$ Also, Nepp and associates have shown that severity of Keratoconjunctivitissicca correlates with severity of diabetic retinopathy. ${ }^{7}$ However in our study, there was no relevant association of diabetic retinopathy with dry eyes.

\section{Conclusion And Recommendations}

Considering increased incidence of dry eyes, early ocular examination in Diabetic patients should be done for early detection of the ocular surface disorders. In order to prevent dry eye syndrome, good glycemic control is important. Examination of dry eyes should be an integral part of the assessment of diabetic eye disease so as to improve the patient's comfort and to prevent or minimize further structural damage to the ocular surface.

\section{References}

[1]. Ghasemi H, Gharebaghi R, Heidary F. Diabetes as a possible predisposer forblepharitis. Can J Ophthalmol 2008 Aug;43(4):485

[2]. World Health Organization. Diabetes. Available at: www. researchandmarkets.com/reportinfo.asp?report id=228279 (Accessed August 30, 2010)

[3]. Goebbles M. Tear secretion and tear film function in insulin dependent diabetics. Br J Ophthalmol2000;84:19-21.

[4]. Mathers WD, Lane JA, Sutphin JE, Zimmerman MB. Model for ocular tear film function. Cornea 1996; 15: 110-119

[5]. Moss SE, Klein R, Klein BE. Incidence of dry eye in an older population. Arch Ophthalmol 2004; 122: 369-73

[6]. Seifart U, Strempel I. The dry eye and diabetes mellitus. Ophthalmologe. 1994 Apr;91(2):235-9

[7]. J. Nepp, C. Abela and I. Polzeret al., Is there a correlation between the severity of diabetic retinopathy and keratoconjunctivitissicca?,Cornea 19 (2000), pp. 487-491.

[8]. Hom M, De Land P. Self-reported dry eyes and diabetic history. Optometry. 2006 Nov;77(11):554-8

[9]. Schaumberg DA, Buring JE, Sullivan DA, Dana MR. Hormone replacement therapy and dry eye syndrome. JAMA 2001 Nov 7;286 (17):2114-9

[10]. Gerhard W. Cibis et al. Fundamentals and principles of ophthalmology, Basic \& Clinical Science Course. American Academy of Ophthalmology. Section 2; LEO Publishers; 2004-2005: 309-17

[11]. Moss SE, Klein R, Klein BE. Prevalence of and risk factors for dry eye syndrome. Arch Ophthalmol 2000;118:1264-8

[12]. Kaiserman I, Kaiserman N, Nakar S, Vinker S. Dry eye in diabetic patients. Am J Ophthalmol 2005;139: 498-503.

[13]. GilbardJP Human tear film electrolyte concentrations in health and dry-eye disease. IntOphthalmolClin. 1994;3427-36

[14]. Schultz RO, Van Horn DL, Peters MA, et al. Diabetic keratopathy. Trans Am OphthalmolSoc 1981;79:180-99.

[15]. Myron Yanoff, Jay S Duker Mosby, Ophthalmology. Second Edition, Mosby; 520-526.

[16]. Khuwaja A.K, Rafique G, White F, Azam S.I. Macrovascular complications and their associated factors among persons with type 2 diabetes in Karachi, Pakistan, a multi-center study. J Pak Med Assoc 2004:54:60-6.

[17]. Dogru M, Katakami C, Inoue M. Tear function and ocular surface changes in noninsulin dependent diabetes mellitus. Ophthalmology 2001, 108: 586 - 91.

[18]. Nichols KK et al. Frequency of dry eye diagnostic test procedures used in various modes of ophthalmic practice. Cornea. 2002; 21:578-583

[19]. Figueroa-Ortiz LC, Jiménez Rodríguez E, García-Ben A, García-Campos J.Sociedad Española de Oftalmología (2010); Elsevier Espana. 